\title{
Differential effectiveness of ARB plus CCB therapy and high-dose ARB therapy in high-risk elderly hypertensive patients: Subanalysis of the OSCAR study
}

\author{
Shokei Kim-Mitsuyama ${ }^{1}$, Hisao Ogawa ${ }^{2}$, Kunihiko Matsui ${ }^{3}$, Tomio Jinnouchi ${ }^{4}$, Hideaki Jinnouchi ${ }^{4}$ \\ and Kikuo Arakawa ${ }^{5}$ for the OSCAR Study Group ${ }^{6}$
}

The OSCAR study was a multicenter prospective randomized study that examined the relative benefit of combined ARB (olmesartan $20 \mathrm{mg}$ per day) plus calcium channel blocker (CCB) therapy vs. high-dose ARB monotherapy (olmesartan $40 \mathrm{mg}$ per day) for prevention of cardiovascular events in elderly Japanese hypertensive patients. The present subanalysis of patients enrolled in the OSCAR study $(n=1078)$ was performed to assess whether baseline eGFR coupled with cardiovascular disease (CVD) could predict the relative benefit of these two treatments. Patients with baseline CVD $(n=769)$ and patients without baseline CVD ( $n=309$ ) were divided into two groups based on baseline eGFR; (i) patients with eGFR of $<60 \mathrm{ml} \mathrm{min}^{-1}$ $1.73 \mathrm{~m}^{-2}$ and (ii) those with eGFR of $\geqslant 60 \mathrm{ml} \mathrm{min}-11.73 \mathrm{~m}^{-2}$. There was a significant treatment-subgroup interaction among these four subgroups in relation to the incidence of primary outcome events $(P=0.007$ for interaction). In patients with CVD and with eGFR of $<60 \mathrm{ml} \mathrm{min}-11.73 \mathrm{~m}^{-2}$, ARB plus CCB therapy was associated with a lower incidence of primary events than high-dose ARB therapy and the difference of the relative risk was statistically significant (hazard ratio: $3.525,95 \%$ confidence interval (CI): 1.676-7.412, $P<0.001$ ). The greater benefit of ARB plus CCB therapy vs. high-dose ARB therapy in this subgroup was associated with less visit-to-visit variability of systolic BP and diastolic BP. In conclusion, baseline eGFR coupled with baseline CVD seems to be a predictor of the relative efficacy of ARB plus CCB therapy vs. high-dose ARB therapy in the elderly hypertensive patients. ARB plus CCB therapy appears to be superior to high-dose ARB therapy for preventing cardiovascular events in the patients with CVD and with eGFR of $<60 \mathrm{ml} \mathrm{min}^{-1} 1.73 \mathrm{~m}^{-2}$.

Hypertension Research (2015) 38, 199-207; doi:10.1038/hr.2014.164; published online 4 December 2014

Keywords: combination; CVD; eGFR; high-dose ARB

\section{INTRODUCTION}

There is accumulating evidence ${ }^{1}$ that ACE inhibitors and ARBs not only reduce blood pressure (BP), but also have pleiotropic effects such as suppression of cardiovascular remodelling, a renoprotective effect, improvement of insulin sensitivity and reduction in onset of diabetes. ${ }^{2}$ In addition, RAS inhibition is a useful therapeutic strategy for heart failure $^{3,4}$ post-myocardial infarction ${ }^{3}$ or diabetic nephropathy. ${ }^{5,6}$ Interestingly, compared with low-dose ARB therapy, high-dose ARB therapy is more effective at delaying the progression of nephropathy in diabetic patients ${ }^{6,7}$ and it reduces the incidence of cardiovascular events further in heart failure patients, ${ }^{8}$ as well as reducing the BP more in hypertensive patients. Thus, uptitration of ARB dosages (high-dose ARB therapy) is a useful strategy for protection against damage to various target organs. On the other hand, the combination of a RAS blocker with a calcium channel blocker (CCB) is thought to be one of the most beneficial strategies both for BP control and for preventing cardiovascular morbidity and mortality in high-risk

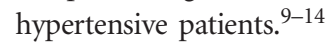

The OlmeSartan and Calcium Antagonists Randomized (OSCAR) study ${ }^{15-17}$ was a multicenter prospective randomized open-label blinded endpoint study that compared ARB plus CCB therapy and high-dose ARB therapy with regard to the prevention of cardiovascular events and death in high-risk elderly Japanese hypertensive patients with baseline cardiovascular disease (CVD) and/or type 2 diabetes. This randomized trial showed that there was no significant difference in the incidence of primary events (fatal and non-fatal cardiovascular events and non-cardiovascular death) between patients receiving ARB plus CCB therapy and those given high-dose ARB therapy. However,

\footnotetext{
${ }^{1}$ Department of Pharmacology and Molecular Therapeutics, Kumamoto University Graduate School of Medical Sciences, Kumamoto, Japan; ${ }^{2}$ Department of Cardiovascular Medicine, Kumamoto University Graduate School of Medical Sciences, Kumamoto, Japan; ${ }^{3}$ Department of Communitiy Medicine, Kumamoto University Hospital, Kumamoto, Japan; ${ }^{4}$ Jinnouchi Clinic, Diabetes Care Center, Kumamoto, Japan and ${ }^{5}$ The Second Department of Internal Medicine, School of Medicine, Fukuoka University, Fukuoka, Japan 6See appendix.

Correspondence: Dr S Kim-Mitsuyama, Department of Pharmacology and Molecular Therapeutics, Kumamoto University Graduate School of Medical Sciences, 1-1-1 Honjyo, Kumamoto 860-8556, Japan.

E-mail: mitsuyam@gpo.kumamoto-u.ac.jp

Received 17 July 2014; revised 26 September 2014; accepted 23 October 2014; published online 4 December 2014
} 
interestingly, the combined $\mathrm{ARB}$ and $\mathrm{CCB}$ therapy reduced the incidence of primary events better than high-dose ARB monotherapy in patients with baseline CVD. ${ }^{17}$ Further planned subanalysis of the OSCAR study has shown that ARB plus CCB therapy also reduces the incidence of primary events more than high-dose $A R B$ therapy in patients with chronic kidney disease (CKD) (eGFR $<60 \mathrm{ml} \mathrm{min}^{-1}$ $\left.1.73 \mathrm{~m}^{-2}\right) .{ }^{15}$ However, the relative impact of baseline CVD vs. baseline eGFR on the efficacy of ARB plus CCB therapy or high-dose ARB therapy in elderly hypertensive patients remains to be elucidated. Moreover, it is unknown whether the superiority of ARB plus CCB therapy over high-dose ARB therapy in patients with baseline $\mathrm{CVD}^{17}$ is independent of baseline eGFR or not. To address these issues, the patients enrolled in the OSCAR study were divided into four groups (CVD-eGFR subgroups); (i) the patients with baseline CVD and low eGFR, (ii) those with baseline CVD and high eGFR, (iii) those without CVD and with low eGFR, (iv) those without CVD and with high eGFR. We investigated the influence of baseline CVD coupled with baseline eGFR on the relative efficacy of ARB plus CCB therapy vs. high-dose ARB therapy in high-risk elderly hypertensive patients.

\section{METHODS}

\section{Patients and procedure of the OSCAR study}

The rationale, study design, treatment protocol, organization and management of the OSCAR study have been reported previously. ${ }^{16}$ In brief, the OSCAR study was a multicenter prospective randomized open-label blinded endpoint study of 1164 elderly Japanese patients (aged 65-84 years) with hypertension. The patients who were eligible had CVD (cerebrovascular disease, cardiac disease, peripheral vascular disease or renal dysfunction) and/or type 2 diabetes at baseline, and inadequate BP control (systolic BP $\geqslant 140 \mathrm{~mm} \mathrm{Hg}$ and/or diastolic BP $\geqslant 90 \mathrm{~mm} \mathrm{Hg}$ ) by olmesartan monotherapy at a dose of $20 \mathrm{mg}$ per day (the standard dose in Japan). In the run-in period, all patients received olmesartan monotherapy at $20 \mathrm{mg}$ per day. If the target BP $(<140 / 90 \mathrm{~mm} \mathrm{Hg})$ was not achieved by olmesartan monotherapy ( $20 \mathrm{mg}$ per day) and it was well tolerated, patients were randomized to one of two treatment arms and received either (i) double the dose of olmesartan ( $40 \mathrm{mg}$ per day: high-dose ARB monotherapy) or (ii) a CCB (amlodipine or azelnidipine) in addition to olmesartan at $20 \mathrm{mg}$ per day (ARB plus CCB therapy). If further treatment was required to achieve the target $\mathrm{BP}$, other antihypertensive drugs such as diuretics or beta-blockers, could be added, but additional ARBs, angiotensin-converting enzyme inhibitors, or CCBs were prohibited. The follow-up period was 3 years. This study was conducted according to the Declaration of Helsinki, and was approved by the Institutional Review Board at each participating site. All patients gave written informed consent.

\section{Endpoints}

The primary endpoint of the OSCAR study was the time to the first event. ${ }^{16}$ The primary endpoint events were fatal and non-fatal cardiovascular events, including cerebrovascular disease (cerebral infarction, cerebral hemorrhage, subarachnoid hemorrhage, unspecified stroke and transient ischemic attacks), coronary artery disease (sudden death, myocardial infarction, angina pectoris and asymptomatic myocardial ischemia), heart failure, other arteriosclerotic diseases (aortic aneurysm, aortic dissection and atherosclerotic diseases), as well as diabetic microvascular complications (neuropathy, retinopathy and nephropathy), deterioration of renal function (doubling of serum creatinine or end-stage renal disease as defined by initiation of hemodialysis or renal transplantation) and non-cardiovascular death.

The secondary endpoints were the incidence of each cardiovascular event, BP changes during follow-up and serious adverse events other than the primary endpoints. The Endpoint Committee assessed all cardiovascular events and deaths without knowledge of the treatment.

\section{Subgroup analysis according to baseline CVD and eGFR} (CVD-eGFR subgroup analysis)

The subgroups with and without CVD at baseline were divided into two groups based on baseline eGFR: (i) patients with eGFR of $<60 \mathrm{ml} \mathrm{min}^{-1} 1.73 \mathrm{~m}^{-2}$

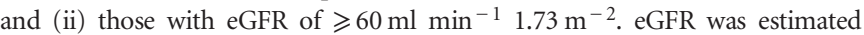
by using the new three-variable Japanese equation. ${ }^{18}$

\section{Visit-to-visit variability of $\mathrm{BP}$}

Visit-to-visit variability of the systolic BP or diastolic BP during the follow-up period in patients receiving ARB plus CCB therapy or high-dose ARB therapy in the above-mentioned four CVD-eGFR subgroups was analyzed and defined by using the $\mathrm{SD}$ or the coefficient of variation $(\mathrm{CV}=\mathrm{SD} /$ mean $)$.

\section{Statistical analysis}

The sample size and power of the study were estimated as described previously. ${ }^{16}$ Primary analyses were performed on an intention-to-treat basis. For the primary endpoints, we compared the ARB plus CCB and high-dose ARB groups using the log-rank test with stratification by gender, age, baseline CVD and type 2 diabetes. Using a stratified Cox proportional hazards model, the hazard ratio (HR) and 95\% confidence interval (CI) were calculated for each treatment group. For each subgroup, time to first event curves were estimated by the Kaplan-Meier method. Repeated measures analysis of variance was used to compare BP between the groups during the follow-up period, and the least squares method was employed to estimate the mean difference of BP between the groups. Changes of eGFR were compared between groups with the unpaired $t$-test adjusted by Holm's method to avoid multiplicity at multiple time points. To estimate the heterogeneity of HRs for CVD-eGFR category as joint confounding, the interaction between treatment groups and CVD-eGFR subgroups was assessed by using the interaction terms in a stratified Cox proportional hazards model. Visit-to-visit variability of BP was compared between groups with the unpaired $t$-test.

In addition to the above subanalysis, we performed three sets of sensitivity analyses. For these analyses, patients with or without baseline CVD were dichotomized into groups at the following eGFR values: (i) eGFR $=65 \mathrm{ml}$

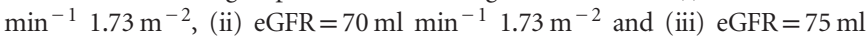
$\min ^{-1} 1.73 \mathrm{~m}^{-2}$

For all analyses, significance was accepted at $P=0.05$ and two-sided tests were used.

\section{RESULTS}

Baseline characteristics of patients by each category in CVD-eGFR subgroups

The number of patients originally enrolled in the OSCAR study was $1164,{ }^{17}$ but 86 patients without serum creatinine values were excluded from the present analysis. Therefore, 1078 patients (the same cohort as used in our previous subanalysis of $\mathrm{CKD})^{15}$ were investigated in the present study. The baseline characteristics of these patients were similar to those of the total patient population of the OSCAR study. The patients with baseline CVD $(n=769)$ or without baseline CVD ( $n=309$ ) were each divided into two groups: (i) patients with eGFR of $<60 \mathrm{ml} \mathrm{min}^{-1} 1.73 \mathrm{~m}^{-2}$ and (ii) those with eGFR of $\geqslant 60 \mathrm{ml} \mathrm{min}^{-1}$ $1.73 \mathrm{~m}^{-2}$. The group with eGFR of $<60 \mathrm{ml} \mathrm{min}^{-1} 1.73 \mathrm{~m}^{-2}$ was designated as the CKD group and the group with eGFR of $\geqslant 60 \mathrm{ml}$ $\min ^{-1} 1.73 \mathrm{~m}^{-2}$ was designated as the non-CKD group.

Tables 1 and 2 show the baseline demographic and clinical characteristics of patients by each category in CVD-eGFR subgroups. Among patients with CVD and with CKD (eGFR of $<60 \mathrm{ml} \mathrm{min}^{-1}$ $1.73 \mathrm{~m}^{-2}$ ), baseline characteristics were well balanced between those receiving high-dose $A R B$ therapy and $A R B$ plus $C C B$ therapy, except for a slight difference of BMI, and the individual baseline CVD were not significantly different between the two treatments. Among patients with CVD and without CKD (eGFR of $\geqslant 60 \mathrm{ml} \mathrm{min}^{-1} 1.73 \mathrm{~m}^{-2}$ ), baseline characteristics were also similar between the two treatments, 


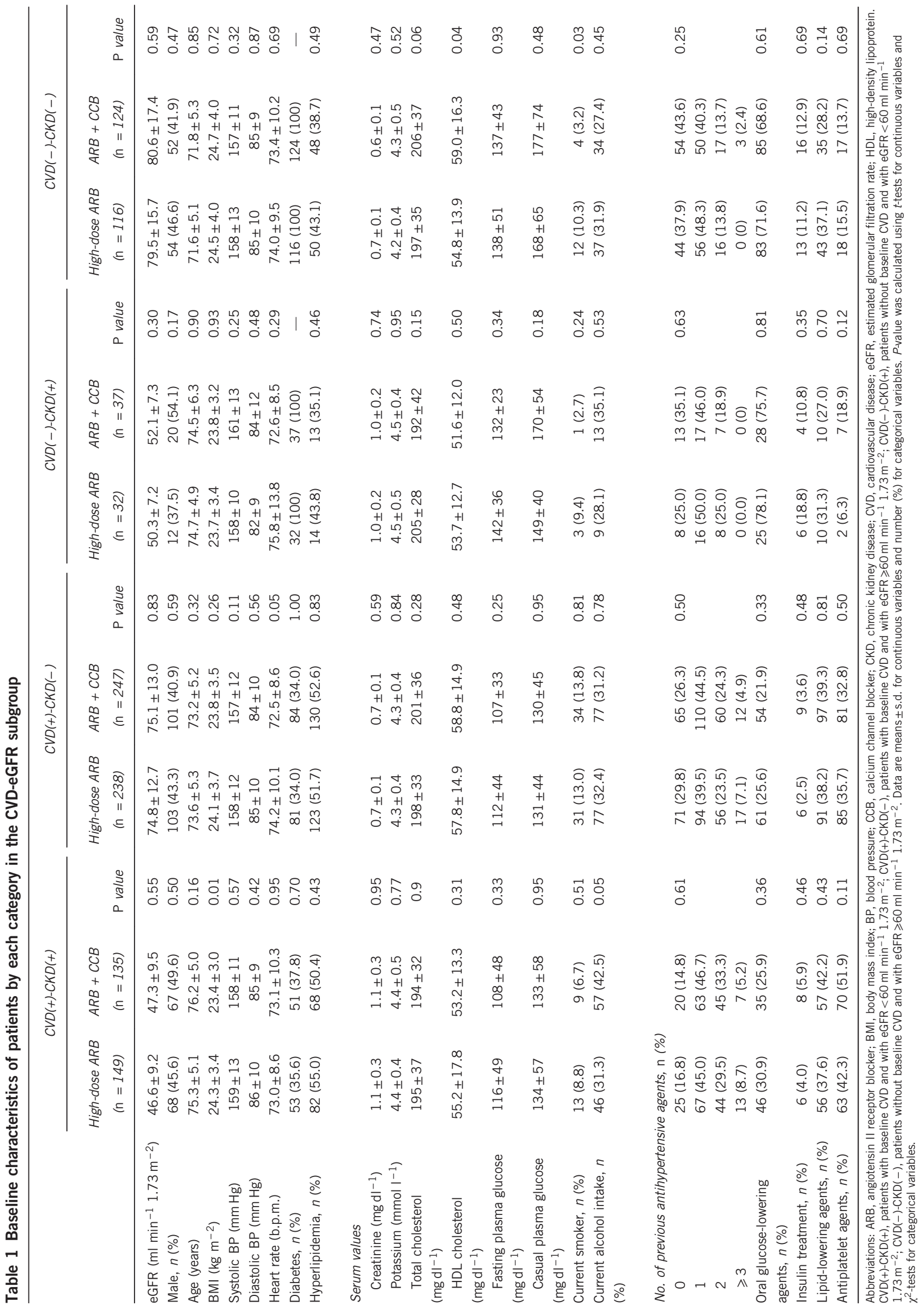


Table 2 Baseline CVD in each subgroup of the patients

\begin{tabular}{|c|c|c|c|c|c|c|}
\hline & \multicolumn{3}{|c|}{$C V D(+)-C K D(+)$} & \multicolumn{3}{|c|}{$C V D(+)-C K D(-)$} \\
\hline & $\begin{array}{l}\text { High-dose ARB } \\
\quad(\mathrm{n}=149)\end{array}$ & $\begin{array}{l}A R B+C C B \\
(\mathrm{n}=135)\end{array}$ & $\mathrm{P}$ value & $\begin{array}{l}\text { High-dose ARB } \\
\quad(\mathrm{n}=238)\end{array}$ & $\begin{array}{l}A R B+C C B \\
(\mathrm{n}=247)\end{array}$ & $\mathrm{P}$ value \\
\hline Stroke, $n(\%)$ & $37(24.8)$ & $37(27.4)$ & 0.62 & $69(29.0)$ & $50(20.2)$ & 0.03 \\
\hline Transient ischemic attack, $n(\%)$ & $16(10.7)$ & $11(8.2)$ & 0.46 & $9(3.8)$ & $16(6.5)$ & 0.18 \\
\hline Asymptomatic cerebrovascular disease, $n(\%)$ & $33(22.2)$ & $25(18.5)$ & 0.45 & $68(28.6)$ & $70(28.3)$ & 0.96 \\
\hline Heart failure, $n(\%)$ & $17(11.4)$ & $17(12.6)$ & 0.76 & $21(8.8)$ & $28(11.3)$ & 0.36 \\
\hline Left ventricular hypertrophy, $n(\%)$ & $32(21.5)$ & $24(17.8)$ & 0.43 & $64(26.9)$ & $69(27.9)$ & 0.80 \\
\hline Aortic aneurysm, $n(\%)$ & $1(0.7)$ & $2(1.5)$ & 0.50 & $0(0.0)$ & $1(0.4)$ & 0.33 \\
\hline $\begin{array}{l}\text { Arteriosclerotic peripheral arterial occlusive disease, } \\
n(\%)\end{array}$ & $5(3.4)$ & $5(3.7)$ & 0.87 & $4(1.7)$ & $10(4.1)$ & 0.12 \\
\hline
\end{tabular}

Abbreviations: ARB, angiotensin II receptor blocker; CCB, calcium channel blocker; CKD, chronic kidney disease; CVD, cardiovascular disease.

$\mathrm{CVD}(+)$-CKD(+), patients with baseline CVD and with eGFR $<60 \mathrm{ml} \mathrm{min}{ }^{-1} 1.73 \mathrm{~m}^{-2} ; \mathrm{CVD}(+)-\mathrm{CKD}(-)$, patients with baseline CVD and with eGFR $\geqslant 60 \mathrm{ml}$ min ${ }^{-1} 1.73 \mathrm{~m}^{-2}$. $P$-value was calculated using $\chi^{2}$-tests.

\begin{tabular}{|c|c|c|c|c|c|c|c|c|c|}
\hline & ber of patier & ts with eve & \multirow[b]{3}{*}{ HRs $(95 \% \mathrm{Cl})$} & \multirow{2}{*}{\multicolumn{4}{|c|}{$\begin{array}{c}\text { Favors } \\
\text { ARB } \\
\stackrel{+}{+} B\end{array}$}} & \multirow[b]{3}{*}{ P Value } & \multirow[b]{3}{*}{ Interaction $\mathbf{P}$} \\
\hline & \multirow{2}{*}{$\begin{array}{c}\text { High-dose } \\
\text { ARB }\end{array}$} & \multirow{2}{*}{$\begin{array}{c}\text { ARB } \\
\stackrel{+}{C} \\
\text { C }\end{array}$} & & & & & & & \\
\hline & & & & 0.01 & 0.10 & 1.0 & 10 & & \\
\hline CVD(+)-CKD(+) & 29(149) & $10(135)$ & $3.525(1.676-7.412)$ & & & & & $<0.001$ & \\
\hline CVD(+)-CKD(-) & $21(238)$ & $24(247)$ & $0.937(0.520-1.689)$ & & & - & & 0.83 & $P=0.007$ \\
\hline CVD(-)-CKD(+) & $1(32)$ & $6(37)$ & $0.204(0.024-1.726)$ & & + & - & & 0.11 & \\
\hline $\operatorname{CVD}(-)-\operatorname{CKD}(-)$ & $6(116)$ & $8(124)$ & $0.767(0.266-2.213)$ & & & 1 & & 0.62 & \\
\hline
\end{tabular}

Figure $1 \mathrm{HRs}$ and $95 \% \mathrm{Cls}$ for incidence of primary outcome events in the CVD-eGFR subgroup. The HRs and 95\% Cls were derived from a stratified Cox proportional hazards model taking into account sex, age and baseline CVD and type 2 diabetes. The $P$ values were derived from a log-rank test, stratified by sex, age and baseline CVD and type 2 diabetes. CVD(+)-CKD(+), patients with baseline CVD and eGFR of $<60 \mathrm{ml} \mathrm{min}^{-1} 1.73 \mathrm{~m}^{-2} ; \mathrm{CVD}(+)-\mathrm{CKD}(-)$, patients with baseline CVD and eGFR of $\geqslant 60 \mathrm{ml} \mathrm{m^{-1 }} 1.73 \mathrm{~m}^{-2} ; \operatorname{CVD}(-)-C K D(+)$, patients without baseline CVD and with eGFR of $<60 \mathrm{ml}$ min ${ }^{-1}$ $1.73 \mathrm{~m}^{-2}$; CVD(-)-CKD(-), patients without baseline CVD and with eGFR of $\geqslant 60 \mathrm{ml} \mathrm{min}^{-1} 1.73 \mathrm{~m}^{-2}$; ARB, angiotensin II receptor blocker; CCB, calcium channel blocker; $\mathrm{Cl}$, confidence interval; $\mathrm{HR}$, hazard ratio.

except for a slight difference of heart rate, and the individual baseline CVD factors were similar between the two treatments apart from a slight difference of previous stroke. Among patients without CVD and with $\mathrm{CKD}$, all baseline characteristics were similar between the highdose ARB and ARB plus CCB groups. Among patients without CVD and without $\mathrm{CKD}$, baseline characteristics were also similar between the two treatments, except for slight differences of some parameters.

\section{Incidence of primary outcome events by each category in CVD-eGFR subgroups}

Figures 1 and 2 shows the HRs with 95\% CIs and the Kaplan-Meier curves, respectively, for primary outcome events in (i) patients with CVD and with CKD, (ii) patients with CVD and without CKD, (iii) patients without CVD and with CKD, and (iv) patients without CVD and without CKD and who were treated with high-dose ARB therapy or ARB plus CCB therapy. There was a significant interaction among these four groups of patients with respect to the incidence of primary outcome events and the treatment-by-subgroup interaction was also significant $(P=0.007$ for interaction).

In patients with CVD and with $\mathrm{CKD}, 29 / 149$ patients assigned to high-dose ARB therapy and 10/135 patients assigned to ARB plus CCB therapy experienced primary events and the difference of relative risk between the two groups was statistically significant (HR: 3.525, 95\% CI: $1.676-7.412, P<0.001)$. On the other hand, in patients with CVD and without CKD, there was no significant difference between high-dose ARB therapy and $A R B$ plus $C C B$ therapy with regard to the incidence of primary events $(P=0.83)$.

However, the incidence of primary events did not differ between the two treatment groups in patients without CVD and with CKD $(P=0.11)$ or patients without CVD and without CKD $(P=0.62)$.

Incidence of each secondary endpoint in the patients with CVD and with CKD

Figure 3 displays the HRs and 95\% CIs for individual secondary endpoints in the subgroup with CVD and with CKD. The incidence of fatal and non-fatal cardiovascular events was lower for patients receiving $\mathrm{ARB}$ plus $\mathrm{CCB}$ therapy than for patients receiving highdose ARB therapy (HR: 4.97, 95\% CI: 1.99-12.41, $P=0.0002$ ). Also, the incidence of cerebrovascular disease was significantly lower in the ARB plus CCB group than in the high-dose ARB group (HR: 8.61, 95\% CI: $1.90-39.13, P=0.001)$. 
a

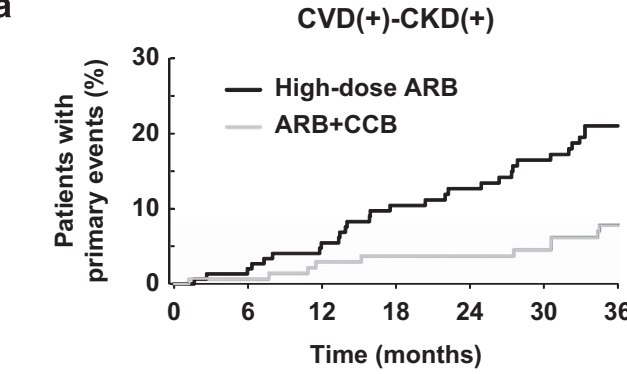

Number at Risk

$\begin{array}{lllllll}\text { High-dose ARB } 149 & 145 & 135 & 124 & 117 & 110 & 104\end{array}$

$\begin{array}{lllllll}A R B & +C C B \\ 135 & 134 & 129 & 123 & 121 & 119 & 114\end{array}$

C

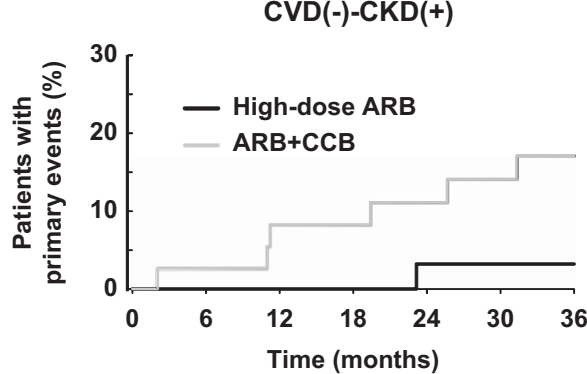

Number at Risk

$\begin{array}{llllllll}\text { High-dose ARB } & 32 & 31 & 31 & 31 & 30 & 30 & 30\end{array}$ b

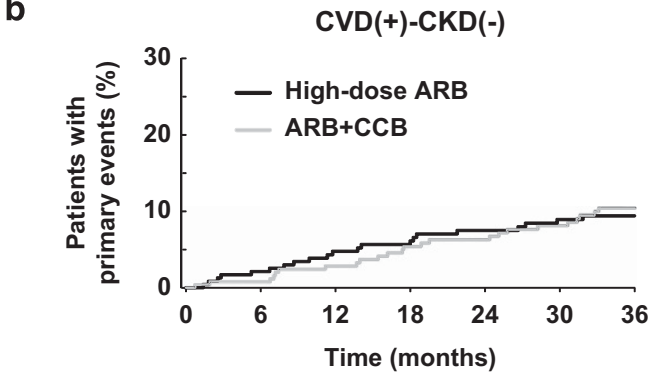

Number at Risk

$\begin{array}{lllllll}\text { High-dose ARB } 238 & 228 & 212 & 205 & 197 & 190 & 188\end{array}$

ARB + CCB $247 \quad 245 \quad 234 \quad 222 \quad 209 \quad 203 \quad 196$

d

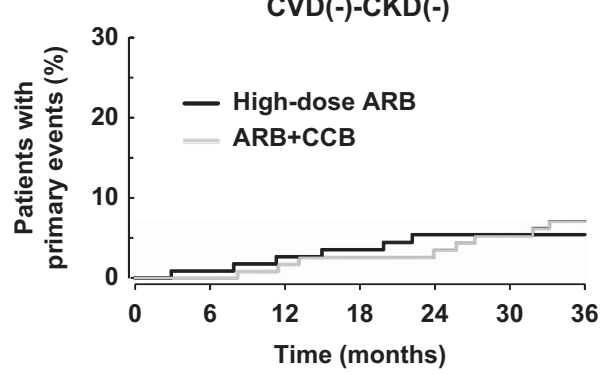

Number at Risk

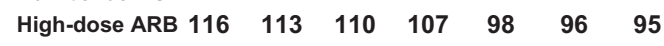

ARB + CCB $124 \quad 123 \quad 116 \quad 115 \quad 109 \quad 105 \quad 103$

Figure 2 Kaplan-Meier curves for primary composite endpoints during the follow-up period in the CVD-eGFR subgroup. (a) CVD(+)-CKD(+) subgroup; (b) CVD $(+)-C K D(-)$ subgroup; (c) CVD (-)-CKD(+) subgroup; (d) CVD( - )-CKD(-) subgroup. Abbreviations used are the same as in Figure 1.

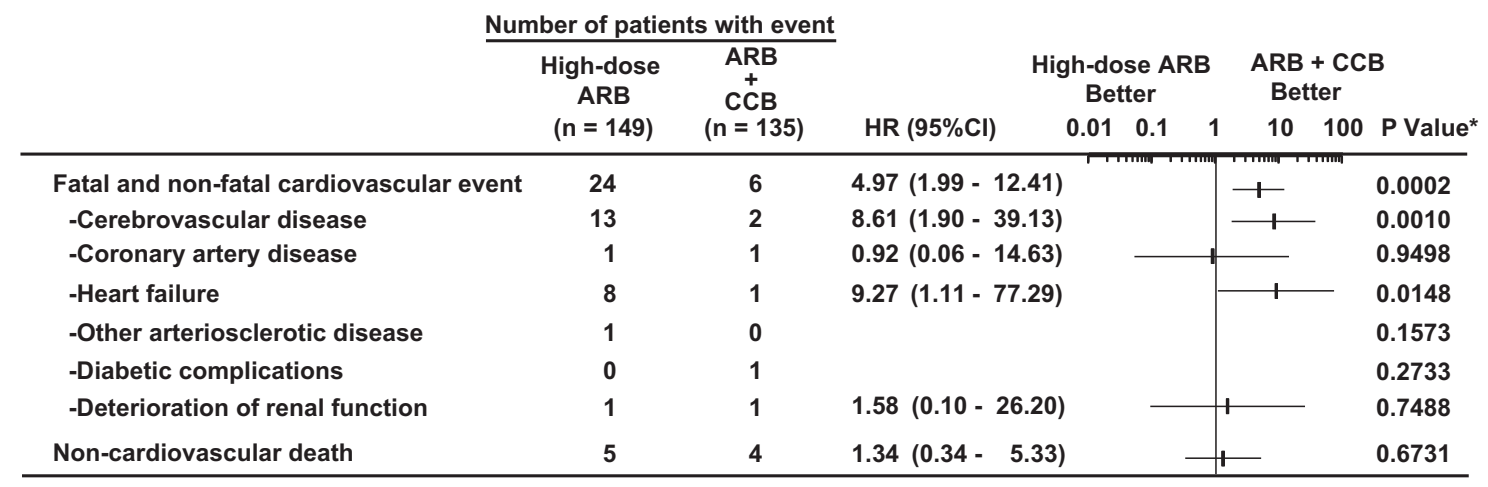

The HRs and $95 \% \mathrm{Cls}$ were derived from stratified Cox proportional hazards model by sex, age, and baseline cardiovascular disease and type 2 diabetes. The $p$ values were derived from a log-rank test, stratified by sex, age, and baseline cardiovascular disease and type 2 diabetes. ${ }^{*} P$ value between high-dose ARB and ARB + CCB.

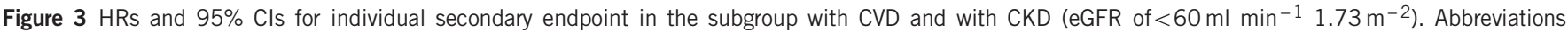
used are the same as in Figure 1.

BP during follow-up

As shown in Supplementary Figure 1A,B in patients with CVD and with $\mathrm{CKD}$, systolic $\mathrm{BP}$ was lower with $\mathrm{ARB}$ plus $\mathrm{CCB}$ therapy than with high-dose $A R B$ therapy and the mean difference of systolic BP throughout the follow-up period was $4.28 \mathrm{~mm} \mathrm{Hg}(P=0.002)$. In patients with CVD and without CKD, the mean difference of systolic $\mathrm{BP}$ between the two treatment groups was not significant $(P=0.063)$, but diastolic BP was lower with ARB plus CCB therapy than with high-dose ARB therapy (mean difference of diastolic BP: $1.88 \mathrm{~mm} \mathrm{Hg}$, $P=0.003)$.
As shown in Supplementary Figure 1C,D in patients without CVD and with $\mathrm{CKD}$, there was no significant difference of systolic or diastolic BP between the two treatment groups during the follow-up period. However, in patients without CVD and without CKD, systolic $\mathrm{BP}$ was lower with $\mathrm{ARB}$ plus $\mathrm{CCB}$ therapy than with high-dose ARB therapy (mean difference of systolic BP: $3.07 \mathrm{~mm} \mathrm{Hg}, P=0.007$ ).

Visit-to-visit variability of systolic and diastolic BP

As shown in Figure 4, patients with CVD and with $\mathrm{CKD}$ receiving ARB plus CCB therapy had a smaller SD-SBP $(P=0.004)$, smaller 
a
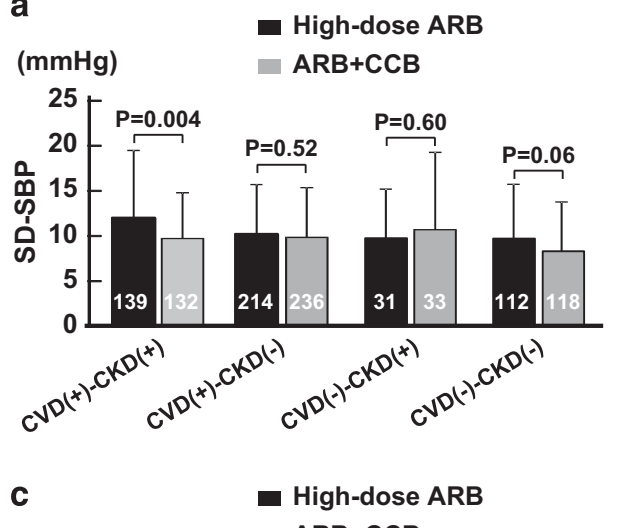

b

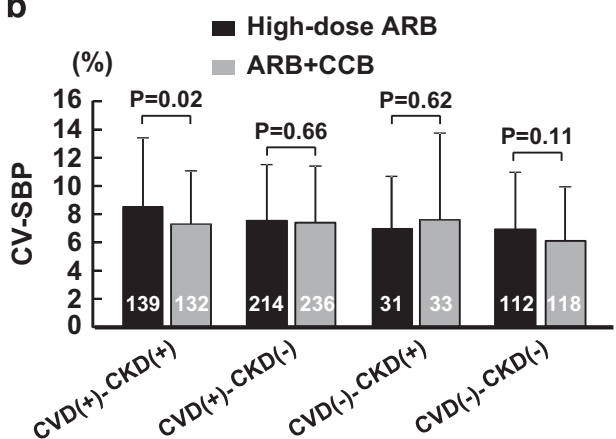

d

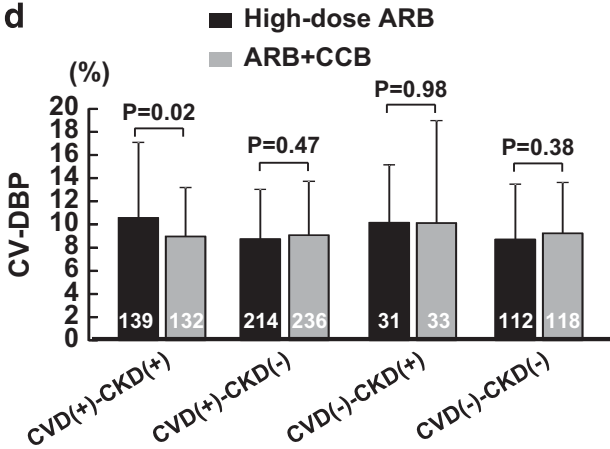

Figure 4 Visit-to-visit variability in systolic and diastolic BP of high-dose ARB group and ARB plus CCB combination group during follow-up period in the CVD-eGFR subgroups. (a) SD-SBP; (b) CV-SBP; (c) SD-DBP; (d) CV-DBP. Abbreviations used are the same as in Figure 1. CV, coefficient of variance; SD, standard deviation. The number within each bar indicates the number of patients in each group. Values are the means \pm s.d.

CV-SBP $(P=0.02)$, smaller SD-DBP $(P=0.004)$, and smaller CV-DBP $(P=0.02)$ than those receiving high-dose ARB therapy. In contrast, the other three subgroups of patients showed no significant differences between ARB plus CCB therapy and high-dose ARB therapy with regard to these four parameters.

\section{Changes of eGFR throughout the follow-up period}

As shown in Supplementary Figure 2, eGFR during follow-up was almost similar between patients receiving ARB plus CCB therapy and high-dose ARB therapy in the four subgroups of patients.

\section{Sensitivity analysis}

In addition to the above-mentioned subanalysis stratified by eGFR value of $<60 \mathrm{or} \geqslant 60 \mathrm{ml} \mathrm{min}^{-1} 1.73 \mathrm{~m}^{-2}$, we performed three sets of sensitivity analyses, in which, patients with or without baseline CVD were categorized according to whether their baseline eGFR was above or below (i) 65, (ii) 70 or (iii) $75 \mathrm{ml} \mathrm{min}^{-1} 1.73 \mathrm{~m}^{-2}$ (see Figures $5 \mathrm{a}-\mathrm{c}$, respectively). In these three sets of analysis, the incidence of primary events was significantly less in ARB plus CCB groups than high-dose ARB group in patients with CVD and lower eGFR, being similar to the results of the original analysis (Figure 1). On the other hand, in all three sets of analysis, high-dose ARB group had less incidence of primary event than ARB plus CCB combination group in patients without CVD and with lower eGFR (eGFR of $<65,70$ or $75 \mathrm{ml} \mathrm{min}^{-1} 1.73 \mathrm{~m}^{-2}$ ).

\section{DISCUSSION}

There is strong evidence that a reduced eGFR is an independent risk factor for CVD and all-cause mortality in a wide range of patient populations, ${ }^{19-21}$ including elderly patients $^{22}$ and hypertensive patients. ${ }^{23}$ An eGFR $<60 \mathrm{ml} \mathrm{min}{ }^{-1} 1.73 \mathrm{~m}^{-2}$ is generally used as the cutoff value for defining $\mathrm{CKD},{ }^{24}$ because it is associated with an increased prevalence of CVD. ${ }^{25,26}$ However, it is unknown whether the baseline eGFR can predict the effectiveness of antihypertensive drugs for preventing cardiovascular events in hypertensive patients. In the present study, patients with or without baseline CVD were categorized by baseline eGFR of $<60$ or $\geqslant 60 \mathrm{ml} \mathrm{min}^{-1} 1.73 \mathrm{~m}^{-2}$. The major findings of the present study were as follows: (i) there was a significant treatment-by-subgroup interaction among the four subgroups; (ii) among patients with CVD and with CKD (that is, an eGFR $<60 \mathrm{ml} \mathrm{min}^{-1} 1.73 \mathrm{~m}^{-2}$ ), the incidence of the primary endpoint was significantly lower when they received ARB plus CCB therapy than with high-dose ARB therapy and this difference was associated with a lower incidence of cerebrovascular disease for combined therapy; and (iii) the lower incidence of endpoint events in patients with CVD and with $\mathrm{CKD}$ receiving $\mathrm{ARB}$ plus $\mathrm{CCB}$ therapy was associated with less $\mathrm{BP}$ variability during the follow-up period relative to that seen with high-dose ARB therapy.

In the OSCAR study, ${ }^{15,17}$ we have previously obtained the findings supporting the concept that baseline CVD or CKD has a significant influence on the relative efficacy of ARB plus CCB therapy and highdose ARB therapy for preventing cardiovascular morbidity and mortality in elderly hypertensive patients. However, the impact of baseline eGFR coupled with previous CVD on the relative efficacy of these two treatments was unclear. To address this issue in the present study, we performed a subgroup analysis of patients with or without baseline CVD who were stratified according to baseline eGFR value of $<60$ or $\geqslant 60 \mathrm{ml} \mathrm{min}^{-1} 1.73 \mathrm{~m}^{-2}$. Interestingly, there was a significant treatment-by-subgroup interaction among the four subgroups of patients. ARB plus CCB therapy was more effective at preventing primary events and cerebrovascular disease than high-dose ARB in patients with CVD and with CKD, whereas there was no significant difference in the incidence of primary events between the two treatments in patients with CVD and without CKD. Therefore, the 
a

\begin{tabular}{|c|c|c|c|c|c|c|c|c|c|}
\hline \multirow{3}{*}{$\frac{u m b e}{h}$} & ber of patien & s with eve & \multirow[b]{3}{*}{ HRs $(95 \% \mathrm{Cl})$} & \multirow{2}{*}{\multicolumn{2}{|c|}{$\begin{array}{c}\text { Favors } \\
\text { High-dose } \\
\text { ARB }\end{array}$}} & \multirow{2}{*}{\multicolumn{2}{|c|}{$\begin{array}{c}\text { Favors } \\
\text { ARB } \\
+\stackrel{+}{C B}\end{array}$}} & \multirow[b]{3}{*}{ P Value } & \multirow[b]{3}{*}{ Interaction $\mathrm{F}$} \\
\hline & \multirow{2}{*}{$\begin{array}{c}\text { High-dose } \\
\text { ARB }\end{array}$} & \multirow{2}{*}{$\begin{array}{l}\text { ARB } \\
\stackrel{+}{C B}\end{array}$} & & & & & & & \\
\hline & & & & 0.01 & 0.10 & 1.0 & 10 & & \\
\hline CVD(+)-eGFR $<65$ & $36(203)$ & $20(194)$ & $1.969(1.135-3.418)$ & & & 1 & & 0.01 & \\
\hline CVD(+)-eGFR $\geqq 65$ & $14(184)$ & $14(188)$ & $1.012(0.479-2.139)$ & & & — & & 0.97 & \\
\hline CVD(-)-eGFR $<65$ & $1(57)$ & $8(61)$ & $0.139(0.017-1.121)$ & & 1 & & & 0.03 & $r=0.01$ \\
\hline CVD(-)-eGFR $\geqq 65$ & 6(91) & $6(100)$ & $0.994(0.319-3.095)$ & & & 1 & & 0.99 & 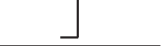 \\
\hline
\end{tabular}

b

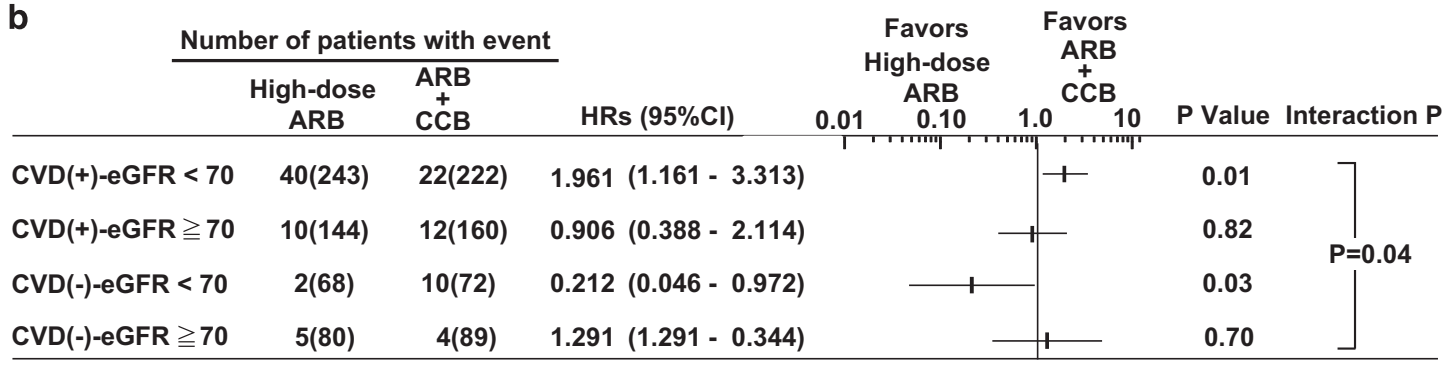

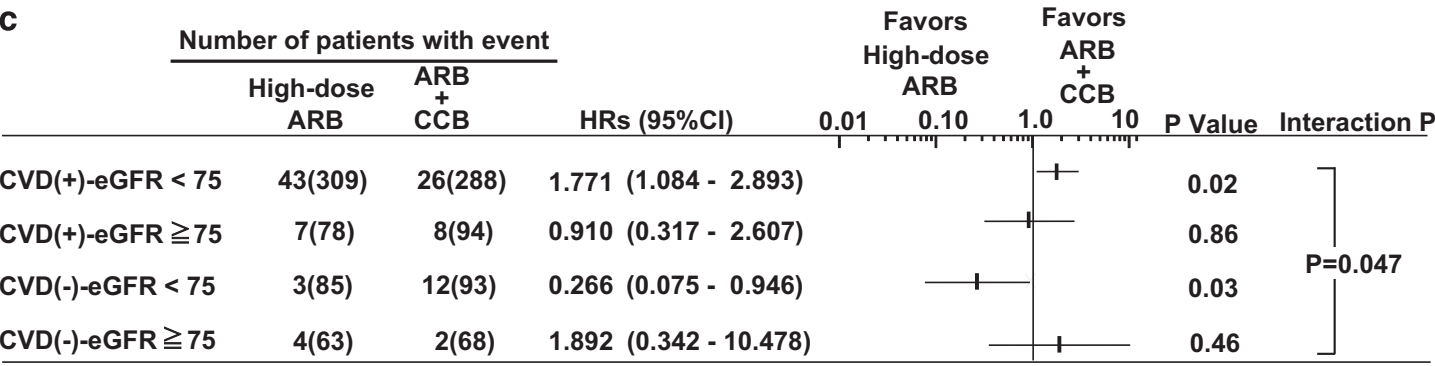

Figure $5 \mathrm{HRs}$ and $95 \% \mathrm{Cls}$ for incidence of primary outcome events in the CVD subgroup with eGFR above or below 65 (a), 70 (b) or 75 (c) $\mathrm{ml}$ min ${ }^{-1}$ $1.73 \mathrm{~m}^{-2}$. The HRs and $95 \% \mathrm{Cls}$ were derived from a stratified Cox proportional hazards model taking into account sex, age and baseline CVD and type 2 diabetes. The $P$ values were derived from a log-rank test, stratified by sex, age and baseline CVD and type 2 diabetes. Abbreviations used are the same as in Figure 1. CVD(+)-eGFR <each reference value (65, 70 and 75), patients with baseline CVD and eGFR<each reference value $\mathrm{ml} \mathrm{min}^{-1} 1.73 \mathrm{~m}^{-2} ; \mathrm{CVD}^{-1}$ $(+)$-eGFR $\geqslant$ each reference value $(65,70$ and 75$)$, patients with baseline CVD and eGFR $\geqslant$ each reference value $\mathrm{ml} \mathrm{min}^{-1} 1.73 \mathrm{~m}^{-2}$; CVD(-)-eGFR $<$ each reference value $(65,70$ and 75$)$, patients without baseline CVD and with eGFR <each reference value $\mathrm{ml} \mathrm{min}^{-1} 1.73 \mathrm{~m}^{-2}$; CVD(-)-eGFR $\geqslant$ each reference value $(65,70$ and 75$)$, patients without baseline CVD and with eGFR $\geqslant$ each reference value $\mathrm{ml} \mathrm{min}{ }^{-1} 1.73 \mathrm{~m}^{-2}$.

baseline eGFR seemed to have a significant influence on the benefit of ARB plus CCB therapy for patients with CVD.

Although there was a significant difference of BP during follow-up between ARB plus CCB therapy and high-dose ARB therapy in patients with CVD and with CKD, it was small in absolute terms (the mean difference of SBP was $4.28 \mathrm{~mm} \mathrm{Hg}$ and that of DBP was $1.39 \mathrm{~mm} \mathrm{Hg}$ ). Accordingly, it is plausible that the difference of mean SBP or DBP between the two treatments during follow-up does not fully explain the significant difference in the incidence of primary events and cerebrovascular disease in patients with CVD and with CKD. Recent analyses ${ }^{27,28}$ indicate that visit-to-visit variability of SBP is a strong predictor of stroke that is independent of the mean SBP value. In addition, increased residual variability of SBP in patients with treated hypertension is associated with a high risk of vascular events. Thus, BP variability seems to have an important role in the progression of organ damage and triggering of vascular events. A systematic review and meta-analysis ${ }^{29}$ found that drug-class effects on BP variability can account for differences in the influence of antihypertensive drugs on the risk of stroke independently of any effect on the mean systolic BP. However, there has been no previous comparison of the effects of ARB plus CCB therapy $v s$. high-dose ARB therapy on $\mathrm{BP}$ variability. In the present study, we analyzed BP variability with these two treatments in four subgroups of patients to examine the potential influence of $\mathrm{BP}$ variability on the relative efficacy of $\mathrm{ARB}$ plus $\mathrm{CCB}$ therapy vs. high-dose ARB therapy in elderly hypertensive patients. We found that ARB plus $\mathrm{CCB}$ therapy was associated with less variability of SBP and DBP than high-dose ARB therapy in patients with CVD and with CKD. These observations suggest that the superiority of ARB plus CCB therapy over high-dose $A R B$ therapy for prevention of primary events and cerebrovascular disease in patients with CVD and with CKD may be at least partially accounted for by better suppression of BP variability.

There were several limitations of this study. First, all three sets of sensitivity analysis (subgroup analysis according to baseline eGFR above or below 65,70 or $75 \mathrm{ml} \mathrm{min}{ }^{-1} 1.73 \mathrm{~m}^{-2}$ ) showed that, among patients without CVD and with low eGFR (that is, eGFR $<$ each reference value), high-dose ARB therapy was associated with a lower incidence of primary events than ARB plus $\mathrm{CCB}$ therapy, in contrast to the superiority of ARB plus CCB therapy for patients with CVD and with low eGFR. In the OSCAR study, all patients without baseline CVD had type 2 diabetes at baseline, because the eligibility for enrolment in the study was dependent on having CVD and/or type 2 diabetes. Renin-angiotensin system is shown to be responsible for the pathophysiology of diabetes and diabetic vascular disease. ${ }^{30,31}$ 
Therefore, it is suggested that the presence of diabetes might have been responsible for the lower incidence of primary events with high-dose ARB therapy than ARB plus CCB therapy in this subgroup. However, the small sample size of the subgroup did not allow us to investigate the potential influence of baseline diabetes on the efficacy of these two treatments. Furthermore, the data are post hoc subgroup analyses. This finding should be considered as hypothesis generating. Second, the change of eGFR during follow-up was similar between the four subgroups of patients receiving either of the two treatments, thereby providing no evidence for an influence of renal function on the incidence of primary events in this study. However, we did not measure urinary albumin excretion, which is a useful predictor of cardiovascular events. ${ }^{32-34}$ Therefore, the precise role of renal function was not elucidated by the present analysis. Finally, it cannot be excluded that the present subanalysis might have no enough power to demonstrate the potential difference between the treatments, although the significant treatment-by-subgroup interaction among the subgroups demonstrated the heterogeneity of treatment effects among subgroups. Further investigation in a larger sample will be required to demonstrate our hypothesis.

In conclusion, the results of the present post hoc subanalysis of patients with or without baseline CVD stratified according to baseline eGFR values suggested that baseline eGFR coupled with baseline CVD may be a useful predictor of the relative effectiveness of $A R B$ plus CCB therapy vs. high-dose ARB therapy in these patients. ARB plus CCB therapy appears to be superior to high-dose ARB therapy for preventing cardiovascular events and cerebrovascular disease in elderly hypertensive patients with CVD and with CKD.

\section{CONFLICT OF INTEREST}

Shokei Kim-Mitsuyama has received consultancy or lecture fees/honoraria from AstraZeneca, Astellas, Boerhinger Ingelheim, Daiichi-Sankyo, Kyowa Hakko Kirin, Novartis, Takeda and Shionogi, and received research grants from AstraZeneca, Astellas, Boerhinger Ingelheim, Daiichi-Sankyo, Kyowa Hakko Kirin, Novartis, Takeda, Shionogi and Mochida. Hisao Ogawa has received research grant from the Japan Heart Foundation for this study, has received consultancy or lecture fees/honoraria from Astellas, AstraZeneca, Bayer, Boehringer Ingelheim, Daiichi-Sankyo, Eisai, Kowa, Kyowa Hakko Kirin, MSD, Novartis, Pfizer, Sanofi-Aventis, Schering-Plough and Takeda, and has received research grants from Astellas, AstraZeneca, Bayer, Boehringer Ingelheim, Daiichi-Sankyo, Kowa, MSD, Novartis, Pfizer, Sanofi-Aventis, Otsuka, Chugai, Bristol-Myers Squibb, Dainippon Sumitomo and Takeda. All other authors declare no conflict of interest.

\section{ACKNOWLEDGEMENTS}

Funding Sources: This work was supported by a grant from the Japan Heart Foundation. Clinical Trial Registration: http://www.clinicaltrials.gov (number NCT00134160).

1 Kim S, Iwao H. Molecular and cellular mechanisms of angiotensin II-mediated cardiovascular and renal diseases. Pharmacol Rev 2000; 52: 11-34.

2 Julius S, Kjeldsen SE, Weber M, Brunner HR, Ekman S, Hansson L, Hua T, Laragh J, Mclnnes GT, Mitchell L, Plat F, Schork A, Smith B, Zanchetti A. Outcomes in hypertensive patients at high cardiovascular risk treated with regimens based on valsartan or amlodipine: the VALUE randomised trial. Lancet 2004; 363: 2022-2031.

3 Pfeffer MA, McMurray JJ, Velazquez EJ, Rouleau JL, Kober L, Maggioni AP, Solomon SD, Swedberg K, Van de Werf F, White H, Leimberger JD, Henis M, Edwards S, Zelenkofske S, Sellers MA, Califf RM. Valsartan, captopril, or both in myocardial infarction complicated by heart failure, left ventricular dysfunction, or both. N Engl J Med 2003; 349: 1893-1906.

4 Pfeffer MA, Swedberg K, Granger CB, Held P, McMurray JJ, Michelson EL, Olofsson B, Ostergren J, Yusuf S, Pocock S. Effects of candesartan on mortality and morbidity in patients with chronic heart failure: the CHARM-Overall programme. Lancet 2003; 362: 759-766.
5 Lewis EJ, Hunsicker LG, Clarke WR, Berl T, Pohl MA, Lewis JB, Ritz E, Atkins RC Rohde R, Raz I. Renoprotective effect of the angiotensin-receptor antagonist irbesartan in patients with nephropathy due to type 2 diabetes. N Engl J Med 2001; 345 . 851-860

6 Parving HH, Lehnert H, Brochner-Mortensen J, Gomis R, Andersen S, Arner P. The effect of irbesartan on the development of diabetic nephropathy in patients with type 2 diabetes. N Engl J Med 2001; 345: 870-878.

7 Hollenberg NK, Parving HH, Viberti G, Remuzzi G, Ritter S, Zelenkofske S, Kandra A Daley WL, Rocha R. Albuminuria response to very high-dose valsartan in type 2 diabetes mellitus. J Hypertens 2007; 25: 1921-1926.

8 Konstam MA, Neaton JD, Dickstein K, Drexler H, Komajda M, Martinez FA, Riegger GA Malbecq W, Smith RD, Guptha S, Poole-Wilson PA. Effects of high-dose versus low dose losartan on clinical outcomes in patients with heart failure (HEAAL study): a randomised, double-blind trial. Lancet 2009; 374: 1840-1848.

9 Jamerson K, Weber MA, Bakris GL, Dahlof B, Pitt B, Shi V, Hester A, Gupte J, Gatlin M Velazquez EJ. ACCOMPLISH Trial Investigators Benazepril plus amlodipine or hydrochlorothiazide for hypertension in high-risk patients. N Engl J Med 2008; 359: 2417-2428.

10 Jamerson KA, Bakris GL, Weber MA. 24-hour ambulatory blood pressure in the ACCOMPLISH trial. N Engl J Med 2010; 363: 98.

11 Jamerson KA, Devereux R, Bakris GL, Dahlof B, Pitt B, Velazquez EJ, Weir M, Kelly RY, Hua TA, Hester A, Weber MA. Efficacy and duration of benazepril plus amlodipine or hydrochlorothiazide on 24-hour ambulatory systolic blood pressure control. Hypertension 2011; 57: 174-179.

12 Shimamoto K, Ando K, Fujita T, Hasebe N, Higaki J, Horiuchi M, Imai Y, Imaizumi T, Ishimitsu $\mathrm{T}$, Ito $\mathrm{M}$, Ito $\mathrm{S}$, Itoh $\mathrm{H}$, Iwao $\mathrm{H}$, Kai $\mathrm{H}$, Kario $\mathrm{K}$, Kashihara $\mathrm{N}$, Kawano $\mathrm{Y}$, Kim-Mitsuyama S, Kimura G, Kohara K, Komuro I, Kumagai H, Matsuura H, Miura K, Morishita R, Naruse M, Node K, Ohya Y, Rakugi H, Saito I, Saitoh S, Shimada K, Shimosawa T, Suzuki H, Tamura K, Tanahashi N, Tsuchihashi T, Uchiyama M, Ueda S Umemura S. Japanese Society of Hypertension Committee for Guidelines for the Management of $\mathrm{H}$. The Japanese Society of Hypertension Guidelines for the Management of Hypertension (JSH 2014). Hypertens Res 2014; 37: 253-387.

13 Mancia G, Fagard R, Narkiewicz K, Redon J, Zanchetti A, Böhm M, Christiaens T, Cifkova R, De Backer G, Dominiczak A, Galderisi M, Grobbee DE, Jaarsma T, Kirchhof P, Kjeldsen SE, Laurent S, Manolis AJ, Nilsson PM, Ruilope LM, Schmieder RE, Sirnes PA, Sleight P, Viigimaa M, Waeber B, Zannad F. Task Force for the management of arterial hypertension of the European Society of Hypertension and the European Society of Cardiology 2013 ESH/ESC Guidelines for the Management of Arterial Hypertension. Blood Press 2013; 22: 193-278.

14 Weber MA, Bakris GL, Jamerson K, Weir M, Kjeldsen SE, Devereux RB, Velazquez EJ, Dahlof B, Kelly RY, Hua TA, Hester A, Pitt B. ACCOMPLISH Investigators Cardiovascular events during differing hypertension therapies in patients with diabetes. $J$ Am Coll Cardiol 2010; 56: 77-85.

15 Kim-Mitsuyama S, Ogawa $\mathrm{H}$, Matsui $\mathrm{K}$, Jinnouchi $\mathrm{T}$, Jinnouchi $\mathrm{H}$, Arakawa $\mathrm{K}$. An angiotensin II receptor blocker-calcium channel blocker combination prevents cardiovascular events in elderly high-risk hypertensive patients with chronic kidney disease better than high-dose angiotensin II receptor blockade alone. Kidney Int 2013; 83: 167-176.

16 Ogawa H, Kim-Mitsuyama S, Jinnouchi T, Matsui K, Arakawa K. Rationale, design and patient baseline characteristics of OlmeSartan and calcium antagonists randomized (OSCAR) study: a study comparing the incidence of cardiovascular events between highdose angiotensin II receptor blocker (ARB) monotherapy and combination therapy of ARB with calcium channel blocker in Japanese elderly high-risk hypertensive patients (ClinicalTrials. gov no. NCT00134160). Hypertens Res 2009; 32: 575-580.

17 Ogawa H, Kim-Mitsuyama S, Matsui K, Jinnouchi T, Jinnouchi H, Arakawa K. OlmeSartan, Calcium Antagonists Randomized Study G. Angiotensin II receptor blocker-based therapy in Japanese elderly, high-risk, hypertensive patients. Am J Med 2012; 125: 981-990.

18 Matsuo S, Imai E, Horio M, Yasuda Y, Tomita K, Nitta K, Yamagata K, Tomino Y, Yokoyama $\mathrm{H}$, Hishida A. Revised equations for estimated GFR from serum creatinine in Japan. Am J Kidney Dis 2009; 53: 982-992.

19 Go AS, Chertow GM, Fan D, McCulloch CE, Hsu CY. Chronic kidney disease and the risks of death, cardiovascular events, and hospitalization. N Engl J Med 2004; 351 1296-1305.

20 Irie F, Iso H, Sairenchi T, Fukasawa N, Yamagishi K, Ikehara S, Kanashiki M, Saito Y, Ota H, Nose T. The relationships of proteinuria, serum creatinine, glomerular filtration rate with cardiovascular disease mortality in Japanese general population. Kidney Int 2006; 69: 1264-1271.

21 Manjunath G, Tighiouart H, Ibrahim H, MacLeod B, Salem DN, Griffith JL, Coresh J, Levey AS, Sarnak MJ. Level of kidney function as a risk factor for atherosclerotic cardiovascular outcomes in the community. J Am Coll Cardiol 2003; 41: 47-55.

22 Manjunath G, Tighiouart H, Coresh J, Macleod B, Salem DN, Griffith JL, Levey AS, Sarnak MJ. Level of kidney function as a risk factor for cardiovascular outcomes in the elderly. Kidney Int 2003; 63: 1121-1129.

23 Ruilope LM, Salvetti A, Jamerson K, Hansson L, Warnold I, Wedel H, Zanchetti A. Renal function and intensive lowering of blood pressure in hypertensive participants of the hypertension optimal treatment (HOT) study. J Am Soc Nephrol 2001; 12: 218-225.

24 Rakugi H, Ogihara T, Umemoto S, Matsuzaki M, Matsuoka H, Shimada K, Higaki J, Ito S, Kamiya A, Suzuki H, Ohashi Y, Shimamoto K, Saruta T, Combination Therapy of Hypertension to Prevent Cardiovascular Events Trial G. Combination therapy for hypertension in patients with CKD: a subanalysis of the Combination Therapy of Hypertension to Prevent Cardiovascular Events trial. Hypertens Res 2013; 36 947-958. 
25 National Kidney Foundation. K/DOQI clinical practice guidelines for chronic kidney disease: evaluation, classification, and stratification. Am J Kidney Dis 2002; 39: S1-266.

26 Levey AS, Bosch JP, Lewis JB, Greene T, Rogers N, Roth D. A more accurate method to estimate glomerular filtration rate from serum creatinine: a new prediction equation. Modification of Diet in Renal Disease Study Group. Ann Intern Med 1999; 130: 461-470.

27 Rothwell PM. Limitations of the usual blood-pressure hypothesis and importance of variability, instability, and episodic hypertension. Lancet 2010; 375: 938-948.

28 Rothwell PM, Howard SC, Dolan E, O'Brien E, Dobson JE, Dahlof B, Sever PS, Poulter NR. Prognostic significance of visit-to-visit variability, maximum systolic blood pressure, and episodic hypertension. Lancet 2010; 375: 895-905.

29 Webb AJ, Fischer U, Mehta Z, Rothwell PM. Effects of antihypertensive-drug class on interindividual variation in blood pressure and risk of stroke: a systematic review and meta-analysis. Lancet 2010; 375: 906-915.

30 Abuissa H, Jones PG, Marso SP, O'Keefe JH Jr. Angiotensin-converting enzyme inhibitors or angiotensin receptor blockers for prevention of type 2 diabetes: a meta-analysis of randomized clinical trials. J Am Coll Cardiol 2005; 46: 821-826.
31 Prasad A, Quyyumi AA. Renin-angiotensin system and angiotensin receptor blockers in the metabolic syndrome. Circulation 2004; 110: 1507-1512.

32 Hillege HL, Fidler V, Diercks GF, van Gilst WH, de Zeeuw D, van Veldhuisen DJ, Gans RO, Janssen WM, Grobbee DE, de Jong PEPrevention of Renal and Vascular End Stage Disease (PREVEND) Study Group. Urinary albumin excretion predicts cardiovascular and noncardiovascular mortality in general population. Circulation 2002: 106: 1777-1782.

33 Ibsen H, Olsen MH, Wachtell K, Borch-Johnsen K, Lindholm LH, Mogensen CE, Dahlof B, Devereux RB, de Faire U, Fyhrquist F, Julius S, Kjeldsen SE, Lederballe-Pedersen O, Nieminen MS, Omvik P, Oparil S, Wan Y. Reduction in albuminuria translates to reduction in cardiovascular events in hypertensive patients: losartan intervention for endpoint reduction in hypertension study. Hypertension 2005; 45: 198-202.

34 Wachtell $\mathrm{K}$, Ibsen $\mathrm{H}$, Olsen MH, Borch-Johnsen K, Lindholm LH, Mogensen CE, Dahlof B, Devereux RB, Beevers G, de Faire U, Fyhrquist F, Julius S, Kjeldsen SE, Kristianson K, Lederballe-Pedersen O, Nieminen MS, Okin PM, Omvik P, Oparil S, Wedel H, Snapinn SM, Aurup P. Albuminuria and cardiovascular risk in hypertensive patients with left ventricular hypertrophy: the LIFE study. Ann Intern Med 2003; 139 : 901-906.

Supplementary Information accompanies the paper on Hypertension Research website (http://www.nature.com/hr)

\section{APPENDIX}

\section{OSCAR Study Group}

Aichi; Kenji Yamada. Akita; Goro Namekawa, Yasushi Suzuki. Aomori; Kenichi Kimura, Morio Aihara. Chiba; Akiko Soyama, Michiko Yonemitsu, Tomotane Shishikura, Toshiyuki Imasawa. Ehime; Masahiro Hasui. Fukuoka; Hidenori Urata, Hiroshi Ikezono, Masahiko Seki, Masaki Munekiyo, Takatoshi Otonari, Tetsuya Ohtsubo, Yasunori Sawayama, Yoichi Hanaoka, Yoshinori Takajo, Yuji Taira. Fukushima; Kuniyoshi Shima. Gifu; Hiroyuki Ohbayashi. Hiroshima; Kazuya Shigenobu. Hokkaido; Chieko Imamoto, Hiromitsu Yokota, Kazuo Yamagata, Kouichi Kanda, Tateo Ogura, Toshio Tsubokura. Hyogo; Akira Kosaka, Akira Tabuchi, Masaharu Shigenobu, Takatoshi Takamiya, Yasuki Makino, Yoshikazu Irie. Kagawa; Hideyasu Kiyomoto, Hirofumi Hitomi. Kagoshima; Yasuhiro Hashiguchi, Yoshihiro Fukuoka, Yoshitaka Shintomi. Kanagawa; Fusahiro Nonaka, Hiroshi Takeda, Masato Nishimura, Nariaki Kanemoto, Takayuki Furuki. Kumamoto; Akira Maki, Akira Sato, Eiichiro Tanaka, Etsuro Tsutsumi, Hajime Shono, Haruo Takeda, Hideaki Jinnouchi, Hirofumi Kann, Hiromi Fujii, Hiroyuki Shono, Hisao Fujimoto, Hisayasu Terazaki, Junichi Matsubara, Kazuhiko Yamada, Kazuhiro Nishigami, Keiichiro Tsuruta, Kenichi Koyama, Kenji Azuma, Koichiro Kataoka, Koji Sasaki, Kouji Honjio, Kunihiro Ohmori, Kunio Idegami, Masakazu Matsukawa, Masamitsu
Toihata, Mikiko Suematsu, Motoko Tanaka, Osamu Hashiguchi, Ryo Fukami, Seiko Fujimoto, Shinichi Uemura, Shiro Mimori, Shojiro Naomi, Shouji Maruta, Shuichi Matsuo, Sunao Kojima, Taiji Sekigami, Takashi Fukunaga, Takashi Kudoh, Takashi Ono, Takeshi Koga, Tomio Wakita, Tomohiro Sawada, Toshihiko Sakanashi, Toshihiro Higashi, Yasuhiro Nagayoshi, Yasuhiro Sakamoto, Yoshihiro Kimura, Yuji Miyao, Yutaka Horio. Kyoto; Ken Takenaka. Miyazaki; Hiroshi Senokuchi, Hirotsugu Ohta, Juniti Miyata, Naoto Yokota, Takeshi Yamamoto. Nagasaki; Hiroyuki Oka, Yoshito Tanioka, Niigata; Toshihide Shu. Okayama; Hirohiko Asonuma, Naoki Kashihara, Naruya Tomita, Takehiko Tokura, Tamaki Sasaki. Osaka; Hidenori Koyama, Katsuo Suyama, Kenei Shimada, Masahito Imanishi, Masanori Emoto, Masayuki Hosoi, Masayuki Nagata, Nobuo Wakaki, Shiro Yanagi, Takao Yoshioka, Takeshi Horio, Tetsuya Hayashi. Saga; Kazuo Moroe, Shiro Hata. Saitama; Hideto Muranaka, Masaru Arai, Shouji Mashiba, Souichirou Ishimoto, Tadahiko Ogasawara, Tomoya Fujino, Tomoyuki Okudaira. Shimane; Yuko Yamane. Shizuoka; Masako Waki. Tokushima; Akira Ota. Kazuto Okagawa, Kenzo Motoki, Takashi Iwase. Tokyo; Akihiko Hachiya, Hiromi Takekawa, Kenzo Matsumura, Masato Yamamoto, Minoru Hojo, Shiho Kaku, Tetsuya Taniguchi, Yasunaga Hiyoshi, Yutaka Shimizu. Yamaguchi; Hideaki Hanamiya. 